\section{Prevalência e fatores associados ao hábito de fumar de gestantes na cidade de São Luís, Maranhão, Brasil}

\section{The prevalence of and factors associated with smoking in pregnant women in the city São Luís, Maranhão, Brazil}

Raphael Lacerda Barbosa 1

Ian Favero Nathasje 2

Deysianne Costa das Chagas 3

Maria Teresa Seabra Soares de Britto e Alves 4

\begin{abstract}
Objectives: to estimate the prevalence and investigate the factors associated with smoking among pregnant women in the city of São Luis, Maranhão.

Methods: a cross-sectional study was carried out with a sample of 5,212 pregnant women attending eleven hospitals in São Luis in 2010. Information on smoking and socio-economic, demographic, reproductive health and lifestyle characteristics were obtained using a standardized questionnaire. The prevalence was estimated and the odds ratio (OR) calculated, with respective confidence intervals of 95\% (C195\%), using a logistic regression model. The data were analyzed using the STATA 12.0 software package.

Results: the prevalence of smoking during pregnancy was $4.1 \%$ (CI95\%:3.55-4.64). The factors associated with smoking were: lack of religion $(O R=1.58 ; C 195 \%: 1.11-2.26) ;$ not having a partner $(O R=1.66 ; C 195 \%: 1.15-2.38)$; having had 2 to 4 births $(O R=2.25$; CI95\%:1.54-3.29) or five births or more $(O R=2.50$; $C 195 \%: 1.23-5.05)$; 0 to 3 prenatal consultations $\quad(O R=2.75 ; \quad C 195 \%: 1.74-4.34)$; economic class $D / E(O R=2.75$; CI95\%:1.22-6.19); and alcohol consumption (OR=7.61; CI95\%:5.5010.55).

Conclusions: the prevalence of smoking was low. There was a reduction in smoking in São Luis from $5.9 \%$, in $1997 / 98$, to $4.1 \%$ in the present study. More emphatic interventions should be directed at pregnant women with the identified risk profile.
\end{abstract}

Key words Pregnancy, Smoking, Alcohol drinking, Religion
1-4 Universidade Federal do Maranhão. Avenida dos Portugueses, 1966. Bacanga. São Luís, MA, Brasil. CEP: 65.080-805.

E-mail: raphael_lacerda.b@hotmail.com

\section{Resumo}

Objetivos: estimar a prevalência e investigar os fatores associados ao hábito de fumar de gestantes na cidade de São Luis, Maranhão.

Métodos: estudo de corte seccional em amostra de 5212 gestantes assistidas em onze hospitais de São Luís em 2010. Informações sobre hábito de fumar e características socioeconômicas, demográficas, de saúde reprodutiva e hábitos de vida foram obtidos utilizando questionário padronizado. Estimativa da prevalência e cálculo de odds ratio (OR), com seus respectivos Intervalos de Confiança de 95\% (IC95\%), foram obtidos em um modelo de regressão logística. Os dados foram analisados no programa estatístico STATA 12.0.

Resultados: a prevalência do hábito de fumar na gravidez foi 4,1\% (IC95\%:3,55-4,64). Os fatores associados ao hábito de fumar foram: ausência de religião (OR=1,58; IC95\%:1,11-2,26); não ter companheiro $(O R=1,66$; IC95\%:1,15-2,38); as categorias 2 a 4 partos $(O R=2,25$; IC95\%:1,54-3,29) e cinco partos ou mais (OR=2,50; IC95\%:1,23-5,05); 0 a 3 consultas pré-natais $(O R=2,75$; IC95\%:1,744,34); classe econômica $D / E(O R=2,75$; IC95\%:1,226,19); e consumo de álcool (OR=7,61; IC95\%:5,5010,55).

Conclusões: a prevalência do hábito de fumar foi baixa. Houve redução do tabagismo em São Luís de 5,9\%, em 1997/98, para 4,1\%, no presente estudo. É necessário que intervenções mais enfáticas sejam dirigidas às gestantes com os fatores de vulnerabilidade encontrados.

Palavras-chave Gravidez, Hábito de fumar, Consumo de bebidas alcoólicas, Religião 


\section{Introdução}

O tabagismo é considerada a principal causa de morte evitável do mundo. ${ }^{1}$ Nas mulheres, o hábito de fumar reduz a fertilidade, aumenta a probabilidade de doenças cardiovasculares e representa riscos à saúde do feto e do recém-nascido, ${ }^{2}$ como a maior chance de baixo peso ao nascer e óbito perinatal.3,4

No Brasil, o hábito de fumar varia entre as regiões e sofre influência de fatores socioculturais e geográficos, com prevalências variando de 14,7\% em Porto Alegre (RS) a 3,6\% em Manaus (AM) entre mulheres maiores de 18 anos. ${ }^{5} \mathrm{Na}$ cidade de São Luís (MA), estudo anterior que avaliou o consumo de tabaco na população gestante encontrou prevalência de $5,9 \% .6$

Alguns fatores comumente associados ao tabagismo durante gravidez são a baixa escolaridade, idade avançada, maior paridade e consumo de álcool.6-10 Por outro lado, o maior número de consultas pré-natais, maior renda familiar e possuir companheiro são exemplos de fatores de proteção.6-8 Outras variáveis, como possuir religião e trabalhar fora de casa, são fatores que também podem influenciar os hábitos de vida, tendo seus efeitos sido investigados em alguns estudos sobre o consumo de tabaco. $6,7,11,12$

A gravidez constitui um período favorável ao desenvolvimento de programas de prevenção e de promoção à saúde, em especial à cessação do hábito de fumar devido ao contato frequente com profissionais da área da saúde e à preocupação da mãe em relação à saúde do seu filho. 8 Considerando que a frequência do hábito de fumar varia de acordo com fatores socioculturais e geográficos, torna-se importante conhecer os fatores associados ao tabagismo das gestantes no âmbito local visando o planejamento de ações de prevenção mais efetivas. ${ }^{7}$

O presente estudo tem o objetivo de estimar a prevalência e investigar os fatores associados ao hábito de fumar de gestantes na cidade de São Luís do Maranhão, em inquérito realizado no ano de 2010 .

\section{Métodos}

Trata-se de um estudo de corte seccional conduzido em 2010 no município de São Luís (MA), Brasil, com base nos dados da coorte de nascimentos BRISA, ${ }^{13,14}$ estabelecida durante o ano de 2010, em duas cidades brasileiras, com o objetivo de testar novas hipóteses para a etiologia da prematuridade.

O tamanho mínimo da amostra foi fixado em 5000 nascimentos, o qual permitiu estimativa de
$50 \%$ de prevalência do agravo (máximo $\mathrm{p} \times \mathrm{q}$ ) com $2 \%$ de precisão e nível de confiança de $99 \%$. O tamanho da amostra também foi adequado para comparar duas proporções, considerando os seguintes parâmetros: de erro tipo I de $5 \%$, poder do estudo de $80 \%$ e produto máximo p x q (assumindo prevalência de $50 \%$ do evento e $50 \%$ de não evento), fixando em $4 \%$ a diferença mínima significativa. Esse produto $(0,5 \times 0,5=0,25)$ estima o tamanho mínimo de amostra com o maior erro possível. Para agravos com prevalência menor que $50 \%$ permitiuse a detecção de diferenças de menor magnitude.

No período de recrutamento da coorte, $98 \%$ dos partos foram hospitalares. Os dados foram coletados em onze maternidades públicas, conveniadas ou particulares que realizaram mais de 100 partos ao ano, totalizando 21.401 nascimentos catalogados no estudo, o que corresponde a $94,7 \%$ dos partos ocorridos em 2010. Destes, um total de 7133 puérperas foram aleatoriamente selecionadas a participar do estudo, sendo aplicados os critérios de exclusão.

A seleção aleatória dos participantes em cada maternidade foi feita mediante amostragem sistemática, após listagem e ordenação dos partos de acordo com a data de ocorrência. Após o sorteio do número inicial de contagem, um em cada três nascimentos foi selecionado para o estudo. A amostra é representativa dos nascimentos ocorridos em São Luís durante o ano de 2010, correspondendo a aproximadamente um terço do total destes nascimentos.

Os dados foram coletados por meio de entrevistas realizadas com as puérperas utilizando questionários padronizados, aplicados por equipe previamente capacitada, preferencialmente nas primeiras 24 horas após o parto.

A variável dependente foi hábito de fumar, definida como fumar um ou mais cigarros por dia. Foram obtidas informações sobre o hábito de fumar nos seis meses que antecederam a gestação atual e durante o primeiro, segundo e terceiro trimestres de gestação. Entre as tabagistas, foram obtidas informações sobre a quantidade diária de cigarros fumados na gravidez atual.

As variáveis de exposição investigadas foram: cor da pele autodefinida (preta, branca), sendo classificadas como mestiça/outras as mulheres que declararam ser de cor/raça "parda/mulata/cabocla/ morena", "amarela/oriental" ou "indígena"; idade $(<20$, entre 20 e 34 e 35 anos ou mais); classe econômica de acordo com o Critério de Classificação Econômica Brasil (CCEB) 15 (classes $\mathrm{A} / \mathrm{B}, \mathrm{C}$ e D/E); escolaridade em anos de estudo (0 a 
4, 5 a 11 e 12 anos ou mais); número de partos (1,2 a 4 e 5 partos ou mais); número de consultas prénatais da gestação atual ( 0 a 3, 4 a 6 e 7 ou mais consultas), situação conjugal (com companheiro e sem companheiro, sendo consideradas com companheiro, as mulheres casadas e em união consensual e sem companheiro, as mulheres solteiras, separadas/desquitadas/divorciadas e viúvas), trabalho fora de casa durante a gestação ( $\operatorname{sim}$ ou não), possuir religião ou frequentar culto ( $\operatorname{sim}$ ou não), consumo de álcool durante a gravidez atual (ingestão de cerveja, vinho, destilados ou outras bebidas alcóolicas).

Os dados foram analisados utilizando o programa estatístico STATA versão 12.0 . Inicialmente realizou-se a descrição da amostra através da distribuição da frequência das características socioeconômicas, demográficas, de saúde reprodutiva e hábitos de vida maternos. A prevalência do hábito de fumar e seus respectivos intervalos de confiança de $95 \%$ (IC95\%) foram estimados e a distribuição de frequência da quantidade de cigarros fumados entre as tabagistas descritas. O teste de McNemar foi utilizado verificar a significância estatística do evento segundo trimestres de gestação. Em seguida, a associação entre o hábito de fumar e as características maternas foi analisada utilizando a técnica da regressão logística. A magnitude da associação foi estimada pelo cálculo dos odds ratio brutos e ajustados e seus respectivos IC95\%. As variáveis independentes (características maternas) associadas ao desfecho a um nível de significância de $p<0,20$ na análise univariada foram incluídas no modelo de regressão múltipla. Permaneceram no modelo final as variáveis independentes com $p<0,10$. Foi considerada significância estatística um valor de $p<0,05$.

Em respeito ao que dispõe a Resolução 196/96 do Conselho Nacional de Saúde (CNS), esta pesquisa foi aprovada pelo Comitê de Ética do Hospital Universitário Presidente Dutra sob o protocolo de número 4771/2008 - 3 .

\section{Resultados}

Dentre os 21.401 nascimentos ocorridos em São Luís no ano de 2010, 7133 mulheres foram aleatoriamente selecionadas para o estudo. Foram excluídas 1682 puérperas por residirem há menos de três meses no município. Das 5451 mulheres elegíveis para a pesquisa, houve a perda de 239 entrevistas devido à recusa ou alta precoce. A amostra final do estudo foi de 5212 mulheres.
A Tabela 1 apresenta as principais características da população de estudo. Mais da metade das gestantes era de cor mestiça/outras $(68,6 \%)$, possuía entre 20 e 34 anos de idade $(73,6 \%)$ e 5 a 11 anos de estudo (80,3\%). Quanto à classe econômica, 50,4\% eram da classe C, seguida pela classe $\mathrm{D} / \mathrm{E}(25,6 \%)$. Em relação à saúde reprodutiva, $47,2 \%$ e $49,2 \%$ das mulheres haviam tido 1 e 2 a 4 partos, respectivamente, e pouco mais de $40 \%(44,2 \%)$ haviam realizado sete ou mais consultas pré-natais durante a gravidez atual. A maioria das gestantes era casada ou possuía união consensual $(80,7 \%)$, não trabalhava fora de casa $(66,4 \%)$ e declarou possuir algum tipo de religião $(81,1 \%)$. Um total de 755 mulheres, que corresponde a $14,5 \%$, referiu ter consumido álcool durante a gestação atual.

A Tabela 2 apresenta a frequência de tabagismo e o número de cigarros fumados na população de estudo. Quanto ao hábito de fumar, 5,6\% das gestantes relataram ter fumado nos seis meses que antecederam a gestação. Um total de 213 gestantes informou hábito de fumar durante a gravidez atual, obtendo-se uma prevalência de 4,1\% (IC95\%: 3,554,64). Em relação à frequência de tabagismo segundo trimestre de gestação, um total de 195 mulheres reportou tabagismo no primeiro trimestre, 119 no segundo e 108 no terceiro trimestre, observando-se prevalências de tabagismo de 3,7\% (IC95\%: 3,2-4,3), 2,3\% (IC95\%: 1,9-2,5) e 2,1\% (IC95\%: 1,7-2,15\%), respectivamente, com redução estatisticamente significante da frequência de tabagismo entre o primeiro e segundo trimestres $(p<0,001)$. A maioria das fumantes $(57,7 \%)$ relatou consumir menos de cinco cigarros por dia.

A Tabela 3 apresenta os resultados das análises brutas e ajustadas da associação entre tabagismo e os fatores de risco investigados. $\mathrm{Na}$ análise bruta, observou-se associação estatisticamente significante do hábito de fumar com ausência de religião $(\mathrm{OR}=1,9$; IC95\%: 1,4-2,6), possuir quatro anos ou menos de estudo $(\mathrm{OR}=2,2$; IC95\%= $1,4-3,4)$, não ter companheiro $(\mathrm{OR}=2,22 ; \mathrm{IC} 95 \%=1,66-2,98)$, antecedentes de dois a quatro partos anteriores à gestação atual $(\mathrm{OR}=2,19$; IC95\%: 1,61-2,97) e 5 ou mais partos $(\mathrm{OR}=3,91$; IC95\%: 2,23-6,84); ter realizado quatro a seis $(\mathrm{OR}=2,28$; IC95\%: $1,56-3,33) \mathrm{e}$ três ou menos consultas pré-natais $(\mathrm{OR}=6,94$; IC95\%=4,70-10,23); classe econômica C (OR: 3,52; IC95\% $=1,69-7,31)$ e classe $\mathrm{D} / \mathrm{E}(\mathrm{OR}=9,83$; IC $95 \%=4,76-20,28)$ e consumo de álcool durante a gestação atual $(\mathrm{OR}=8,68$; IC95\%=6,54-11,52). As gestantes com mais de 35 anos de idade $(\mathrm{OR}=0,41$; IC95\% $=0,04-0,53$ ); com 12 anos ou mais de estudo $(\mathrm{OR}=0,08 ; \mathrm{IC} 95 \%=0,03-0,26)$; e que trabalharam 
Tabela 1

Características socioeconômicas, demográficas, de saúde reprodutiva e hábitos de vida de gestantes de São Luís, Maranhão, 2010.

\begin{tabular}{|c|c|c|}
\hline Variáveis ( $\mathrm{N}=5212$ ) & $\mathbf{N}$ & $\%$ \\
\hline \multicolumn{3}{|l|}{ Cor da pele* } \\
\hline Branca & 961 & 18,5 \\
\hline Preta & 674 & 12,9 \\
\hline Mestiça/Outras & 3573 & 68,6 \\
\hline \multicolumn{3}{|l|}{ Idade (anos) } \\
\hline$<20$ & 961 & 18,5 \\
\hline $20-34$ & 3838 & 73,6 \\
\hline 35 ou mais & 413 & 7,9 \\
\hline \multicolumn{3}{|l|}{ Escolaridade* (anos) } \\
\hline 0 a 4 & 239 & 4,6 \\
\hline 5 a 11 & 4174 & 80,3 \\
\hline 12 anos ou mais & 785 & 15,1 \\
\hline \multicolumn{3}{|l|}{ Classe econômica** } \\
\hline$A-B$ & 939 & 18,0 \\
\hline C & 2624 & 50,4 \\
\hline $\mathrm{D} / \mathrm{E}$ & 1335 & 25,6 \\
\hline Não sabem/sem informação & 314 & 6,0 \\
\hline \multicolumn{3}{|l|}{$N^{\circ}$ de partos* } \\
\hline 1 & 2460 & 47,2 \\
\hline 2 a 4 & 2563 & 49,2 \\
\hline 5 ou mais & 188 & 3,6 \\
\hline \multicolumn{3}{|c|}{$\mathrm{N}^{\circ}$ consultas pré-natais (gestação atual) } \\
\hline 0 a 3 & 657 & 12,6 \\
\hline 4 a 6 & 1989 & 38,2 \\
\hline$\geq 7$ & 2304 & 44,2 \\
\hline Não sabem/sem informação & 262 & 5,0 \\
\hline \multicolumn{3}{|l|}{ Situação conjugal } \\
\hline Com companheiro & 4207 & 80,7 \\
\hline Sem companheiro & 1005 & 19,3 \\
\hline \multicolumn{3}{|l|}{ Trabalho fora de casa } \\
\hline Sim & 1753 & 33,6 \\
\hline Não & 3459 & 66,4 \\
\hline \multicolumn{3}{|l|}{ Religião } \\
\hline Sim & 4226 & 81,1 \\
\hline Não & 986 & 18,9 \\
\hline \multicolumn{3}{|l|}{ Consumo de álcool } \\
\hline Sim & 755 & 14,5 \\
\hline Não & 4457 & 85,5 \\
\hline
\end{tabular}

* Excluídos não sabem/sem informação das variáveis: Cor da pele $(n=4)$; Escolaridade $(n=14)$; No de partos $(n=1)$;

** A variável Classe econômica foi categorizada de acordo com o Critério de Classificação Econômica Brasil (CCEB), sendo reagrupada em três categorias: A/B, C e D/E. 
fora de casa $(\mathrm{OR}=0,43 ; \mathrm{IC} 95 \%=0,30-0,61)$ tiveram menos chance de fumar durante a gravidez.

$\mathrm{Na}$ análise ajustada, os fatores que se mantiveram independentemente associados ao hábito de fumar foram: ausência de religião $(\mathrm{OR}=$ 1,58 ; IC95\%= 1,11-2,26); não ter companheiro $(\mathrm{OR}=1,66 ; \mathrm{IC} 95 \%=1,15-2,38)$; antecedentes de dois a quatro partos anteriores à gestação atual $(\mathrm{OR}=$
2,25 ; IC95\%= $1,54-3,29$ ) e de cinco ou mais partos $(\mathrm{OR}=2,50$; IC $95 \%=1,23-5,05)$; três ou menos consultas pré-natais na gestação atual $(\mathrm{OR}=2,75$; IC95\%= 1,74-4,34); classe econômica $\mathrm{D} / \mathrm{E}(\mathrm{OR}=$ 2,75; IC95\%: 1,22-6,19) e consumo de álcool durante a gestação atual $(\mathrm{OR}=7,61 ; \mathrm{IC} 95 \%=5,50$ 10,55 ) (Tabela 3).

Tabela 2

\begin{tabular}{|c|c|c|c|}
\hline Hábito de fumar ( $\mathrm{N}=5212)$ & $\mathbf{N}$ & $\%$ & IC95\% \\
\hline Fumou 6 meses antes da gestação atual* & 294 & 5,6 & $5,0-6,3$ \\
\hline Fumou na gravidez atual & 213 & 4,1 & $3,6-4,6$ \\
\hline \multicolumn{4}{|l|}{ Quantidade de cigarros fumados por dia** } \\
\hline$<5$ cigarros & 123 & 57,7 & $50,8-64,5$ \\
\hline 5 a 10 cigarros & 45 & 21,1 & $15,9-27,2$ \\
\hline$>10$ cigarros & 27 & 12,7 & $8,5-17,9$ \\
\hline Não sabem/sem informação & 18 & 8,5 & $5,0-13,0$ \\
\hline
\end{tabular}

* Excluído não sabem/sem informação da variável: Fumou 6 meses antes da gestação atual $(n=1)$;

** O denominador para o cálculo de prevalências corresponde a $n=213$ referente às gestantes que fumaram na gravidez atual. 


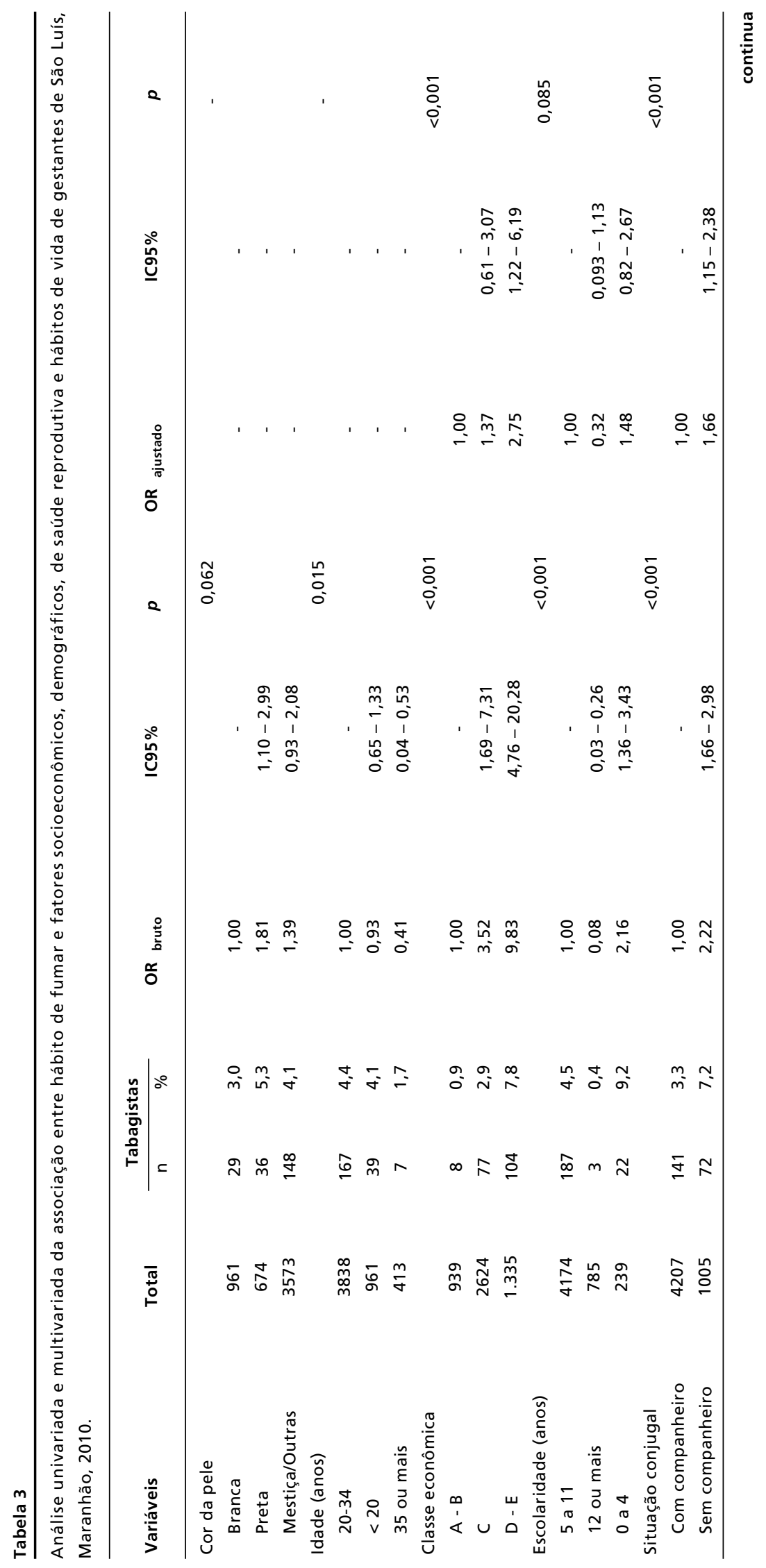




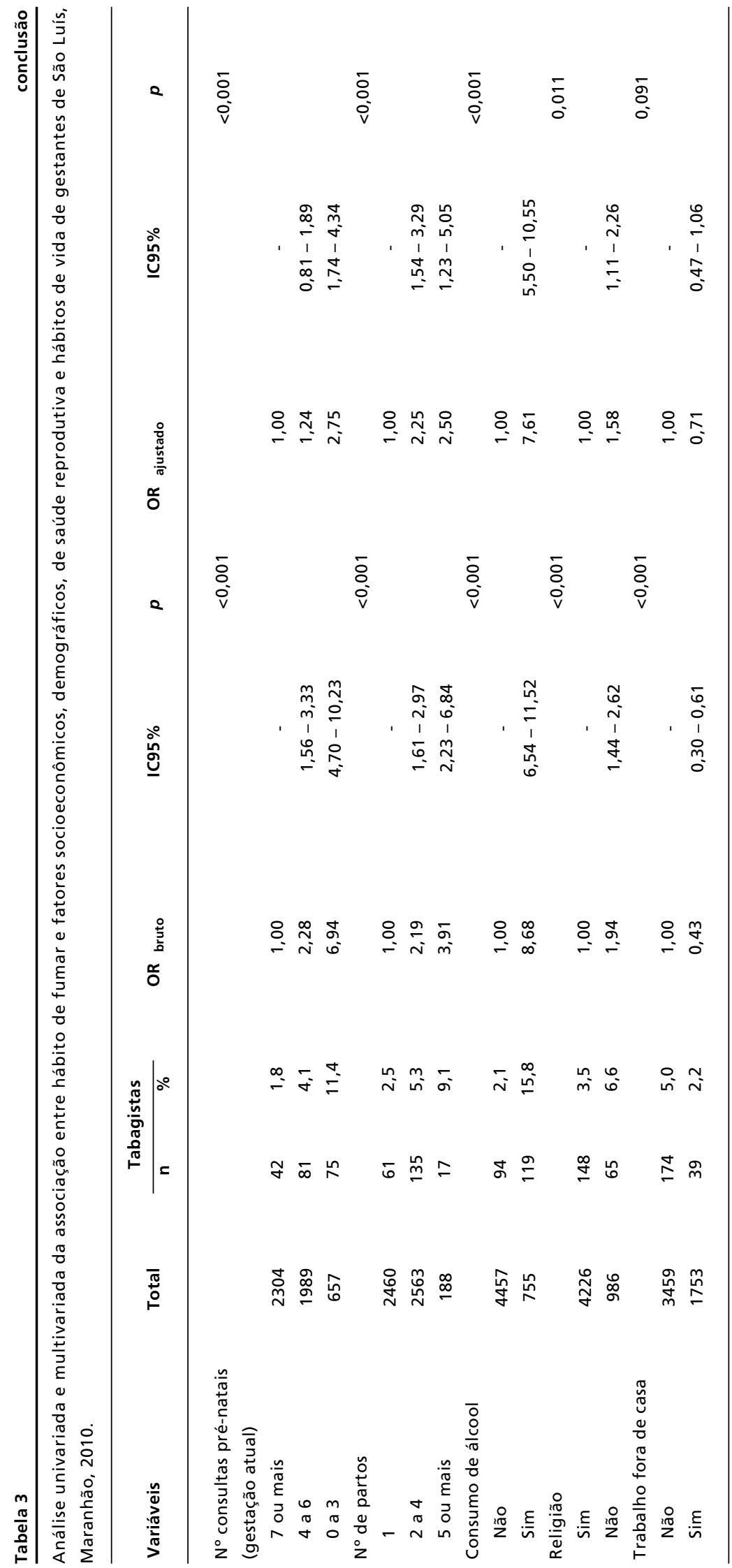




\section{Discussão}

Neste estudo, observou-se que a prevalência do hábito de fumar durante a gravidez foi de $4,1 \%$, apresentando uma progressiva redução da frequência deste hábito ao longo do período gestacional. Essa prevalência de tabagismo entre as gestantes de São Luís (MA) é considerada baixa, quando comparada à encontrada em estudos recentes realizados em outras cidades brasileiras, cujos níveis de prevalência variaram de $23,0 \%$ (Zhang et al. ${ }^{4}$ ), na cidade de Rio Grande (RS), e $21,1 \%$ (Reis et al. ${ }^{10}$ ) na cidade do Rio de Janeiro (RJ). Em estudos realizados na Argentina, Uruguai, Dinamarca, Noruega e Finlândia, o hábito de fumar na gravidez também foi maior, com prevalências de $10,3 \%, 18,3 \%, 12,5 \%$, $16,5 \%$ e $15 \%$, respectivamente. 16,17

Em relação ao Brasil, é interessante destacar a tendência decrescente do hábito de fumar na gravidez na direção Sul-Norte do País.6,7 Kroeff et $a l .7$ avaliaram o tabagismo em gestantes de seis capitais brasileiras nos anos 1991-95, encontrando prevalências de 31,9\% em Porto Alegre (Sul), 16,1\% em São Paulo (Sudeste), 9,2\% em Salvador (Nordeste) e 7,2\% em Manaus (Norte). São Luís apresentou resultado similar às capitais da Região Norte e Nordeste, reforçando a tendência demonstrada anteriormente. Nos dias atuais, verifica-se uma redução da prevalência do tabagismo durante a gravidez em relação ao encontrado décadas atrás. Por exemplo, houve diminuição do hábito de fumar na população gestante em São Luís de 5,9\%, em trabalho realizado em $1997 / 98$, para $4,1 \%$ no presente estudo. 6 Esse resultado pode ser explicado pela diminuição do tabagismo na população geral, devido a um conjunto de estratégias adotadas pelo país contra o consumo de tabaco, como a proibição das propagandas de marcas de cigarro, e pelo crescimento da cobertura pré-natal, permitindo maior acesso das gestante a serviços de saúde. 1,2,8,18,19

Apesar da redução do tabagismo, as características socioeconômicas, demográficas e de saúde reprodutiva das mulheres que fumaram durante a gravidez na década de 1990 se mantiveram semelhantes aos encontrados no estudo atual, permanecendo as gestantes sem companheiro, com maior paridade, menor número de consultas prénatais e baixa renda, o grupo com a maior prevalência de tabagismo. ${ }^{6}$ Isso demonstra que os fatores associados ao hábito de fumar na cidade de São Luís pouco se modificaram nos últimos vinte anos.

A prevalência do tabagismo diminuiu no período que compreendeu os seis meses antecedentes à gestação $(5,6 \%)$, quando comparada à prevalência encontrada no primeiro trimestre $(3,7 \%)$, sugerindo a possibilidade de que a gravidez influencie a gestante a reduzir o seu consumo de tabaco. Esses dados corroboram as evidências fornecidas por estudos que observaram a cessação espontânea do tabagismo em mulheres no início da gravidez, na ausência de qualquer intervenção médica. 10,16,20,21 Além disso, percebeu-se uma redução da frequência do tabagismo ao longo do período gestacional, reforçando a hipótese de que a gravidez favorece a mudança deste hábito comportamental considerado nocivo à gestante.

Adicionalmente, os dados mostraram que a chance de parar de fumar foi maior nos períodos iniciais da gravidez, em relação aos períodos subsequentes, e que as gestantes que mantiveram o hábito depois do segundo trimestre tiveram maior chance de fumar durante toda a gravidez. Isso pode ser explicado porque as gestantes que decidem cessar o tabagismo geralmente possuem menor carga tabágica e param de fumar assim que são notificadas da gravidez, enquanto que aquelas que não param de fumar na fase inicial da gestação consomem mais cigarros por dia e são mais resistentes à redução do tabagismo, tendendo a manter o hábito ao longo da gravidez.10,21,22 Para as mulheres que param de fumar, alguns dos motivos que explicam essa mudança de comportamento são a vontade e a determinação em parar de fumar, pedidos de pessoas próximas, apoio social, familiar e profissional, como também preocupações relacionadas à saúde do bebê ou à gravidez. $8,10,21$

$\mathrm{Na}$ análise ajustada, constatou-se que a ausência de religião foi um fator de risco independentemente associado ao hábito de fumar durante a gravidez. As mulheres que declararam não possuir religião tiveram 58\% mais chance de fumar na gestação, sinalizando para os benefícios da religiosidade sobre hábitos comportamentais nocivos para a população gestante. Não foram encontradas pesquisas mostrando a relação entre religião e tabagismo na gravidez, entretanto, estudos demonstram que a religiosidade é relevante na modulação do uso de drogas lícitas e ilícitas por diversos grupos populacionais. ${ }^{11,23,24}$ Essa associação pode ser evidenciada, por exemplo, em trabalho realizado no Estado de Pernambuco, em que os adolescentes que disseram possuir prática religiosa apresentaram $39 \%$ menos chance de fumar quando comparado aos adolescentes que se declararam sem religião.11 Mais estudos são necessários para reforçar essa associação e identificar os mecanismos pelos quais a religião exerce seu efeito protetor. 
Com relação à situação conjugal, as gestantes solteiras, separadas ou viúvas tiveram mais chance de fumar durante a gravidez, com resultado semelhante ao encontrado na literatura. 7,9,17 Explicações para esse resultado podem ser atribuídas ao fato das gestações em mulheres solteiras estarem frequentemente associadas a outros fatores de risco para comportamentos gestacionais noviços, como baixa escolaridade, baixo nível socioeconômico e gravidez indesejada. $9,17,25$ A falta de suporte social, segurança relacional e outros conflitos pessoais podem também ter importantes contribuições para o evento. 25 Entretanto, apesar da menor probabilidade de fumar das mulheres casadas ou em união consensual, é importante que os profissionais de saúde verifiquem também o hábito de fumar do companheiro. Segundo alguns estudos, as mulheres que possuem companheiros tabagistas têm mais chance de fumar durante a gravidez.6,26,27 Em pesquisa realizada na cidade de Ribeirão Preto (SP), por exemplo, as mulheres com companheiros fumantes tiveram em torno de três vezes mais possibilidade de fumar durante a gestação. 6

Os fatores relacionados à saúde reprodutiva (número de consultas pré-natais e paridade) mantiveram associação com o hábito de fumar na gestação, em compatibilidade com outros estudos.6-8 $\mathrm{Na}$ presente pesquisa, as mulheres que realizaram sete ou mais consultas pré-natais apresentaram prevalência de tabagismo de $1,8 \%$, em comparação aos $11,4 \%$ da categoria com zero a três consultas. É notável pelos resultados a importância do pré-natal durante a gravidez, onde o contato com profissionais de saúde possibilita a orientação e o incentivo a hábitos de vida saudáveis, como a cessação do tabagismo. Essa relação é discutida em outros trabalhos sobre o tema e reafirma o impacto das consultas pré-natais e do contato da equipe de saúde, especialmente do médico obstetra, na melhoria da saúde da mulher.6,8,18

As gestantes pertencentes à classe econômica $\mathrm{D} / \mathrm{E}$ associaram-se estatisticamente com o hábito de fumar na gravidez. Diferente do presente estudo, algumas pesquisas brasileiras que avaliaram o fator econômico categorizaram-no através de renda familiar em salários mínimos.6,28 O Critério de Classificação Econômica Brasil tem a função de estimar o poder de compra das pessoas e famílias urbanas e possui equivalência com a renda média familiar da população brasileira. ${ }^{15}$ Quando comparados, os trabalhos realizados na cidade de Ribeirão Preto (SP), 6 em 1994, e de Pelotas (RS), 28 em 1983-93, encontraram resultados semelhantes ao de São Luís. Esses resultados mostram que, apesar da passagem do tempo, as gestantes de baixa renda continuam sendo um grupo de risco para o consumo de tabaco nos dias atuais. Além disso, em estudo realizado na Finlândia e outros países nórdicos, foi demonstrado uma chance sete a oito vezes maior de fumar nas gestantes do grupo socioeconômico mais baixo, mostrando que essa associação também é encontrada em países desenvolvidos. ${ }^{17} \mathrm{~A}$ menor escolaridade, menor acesso à informação e à serviços de saúde dos indivíduos de baixa renda podem explicar a maior vulnerabilidade desse grupo ao tabagismo. ${ }^{28,29} \mathrm{~A}$ maior prevalência do hábito de fumar nas classes econômicas mais baixas mostra que condutas como o aumento do preço do cigarro tem um impacto populacional importante.1,2,29 Aumentar impostos para encarecer o preço do cigarro é a maneira mais efetiva de reduzir o tabagismo, especialmente nos grupos de baixa renda.1,2 Um aumento do preço do cigarro em torno de $10 \%$ é capaz de reduzir o consumo em países subdesenvolvidos e em desenvolvimento em até $8 \% .1,19$

Um percentual de $14,5 \%$ das gestantes relatou ingestão de bebidas alcoólicas durante a gravidez, tendo se observado uma forte associação entre este fator e o hábito de fumar. Estudo realizado na cidade do Rio de Janeiro, entre 1999 e 2006, encontrou concomitância do consumo de álcool em 31,3\% das gestantes fumantes. ${ }^{9}$ Outros trabalhos na literatura também reforçam essa associação, mostrando a necessidade de que as ações preventivas em álcool e tabaco para as mulheres grávidas devam ser realizadas de maneira integrada. 7,10

As gestantes com menor escolaridade (zero a quatro anos de estudo) não mantiveram associação estatística, na análise ajustada, com o hábito de fumar durante a gravidez. Esse resultado contrasta com a literatura, sendo a baixa escolaridade um fator tradicionalmente associado ao tabagismo. 1,2,6,9,28,29 Entretanto, no estudo prévio realizado na cidade São Luís no biênio de 1997/98, o mesmo resultado foi encontrado, com a categoria zero a quatro anos não mantendo associação na análise ajustada. ${ }^{6}$ Não está claro o porquê da falta de associação, mas a persistência do resultado nos dois estudos demonstra que algum fator local pode ser o responsável pelo achado. Novos estudos são necessários para esclarecer a divergência em relação à escolaridade e propor justificativas para o resultado da pesquisa.

A não realização da dosagem de biomarcadores, como a nicotina no sangue ou na saliva e a cotinina na urina, para confirmar o uso do tabaco, constituiu uma importante limitação do estudo. $\mathrm{O}$ autorrelato sem confirmação bioquímica pode interferir nos resultados, na medida em que, provavelmente por 
sentimentos de culpa e constrangimento, as gestantes podem não fornecer informações verídicas a respeito de seu hábito de fumar. ${ }^{27,30}$ É importante que estudos futuros utilizem parâmetros bioquímicos para aumentar a confiabilidade dos resultados.

Conclui-se que, em São Luís (MA), a prevalência de tabagismo entre as gestantes é baixa, quando comparada a outras localidades do país. O hábito de fumar diminuiu entre as mulheres no início da gravidez, em relação ao período dos seis meses antes gestação. As gestantes solteiras, com baixo nível socioeconômico, pouco contato com serviços pré-natais, maior paridade e que consumiram álcool tiveram mais chance de fumar na gravidez, expondo o binômio materno-fetal às consequências deletérias do tabaco. Possuir religião, uma variável ainda pouco avaliada em estudos sobre uso de drogas lícitas e ilícitas na população gestante, reduziu a

\section{Referências}

1. World Health Organization. WHO report on the global tobacco epidemic, 2013: enforcing bans on tobacco advertising, promotion and sponsorship. Geneva; 2013.

2. Eriksen M, Mackay J, Ross H. The Tobacco Atlas. Fourth Edition. Atlanta, GA: American Cancer Society, New York, NY: World Lung Foundation; 2012.

3. National Center for Chronic Disease Prevention and Health Promotion Office on Smoking and H. Reports of the Surgeon General. The Health Consequences of Smoking-50 Years of Progress: A Report of the Surgeon General. Atlanta (GA): Centers for Disease Control and Prevention (US); 2014.

4. Zhang L, González-Chica DA, Cesar JA, Mendoza-Sassi RA, Beskow B, Larentis N, Blosfeld T. Tabagismo materno durante a gestação e medidas antropométricas do recémnascido: um estudo de base populacional no extremo sul do Brasil. Cadernos de Saúde Pública. 2011; 27: 1768-76.

5. Vigitel Brasil 2013. Vigilância de fatores de risco e proteção para doenças crônicas por inquérito telefônico. Brasília: Ministério da Saúde; 2014.

6. Ribeiro VS, Figueiredo FP, Silva AAM, Batista RLF, Barbieri MA, Lamy Filho F, Alves MTSSB, Bettiol H, Santos AM. Do socioeconomic factors explain why maternal smoking during pregnancy is more frequent in a more developed city of Brazil? Braz J Med Biol Res. 2007; 40: $1203-10$

7. Kroeff LR, Mengue SS, Schmidt MI, Duncan BB, Favaretto ALF, Nucci LB. Fatores associados ao fumo em gestantes avaliadas em cidades brasileiras. Rev Saúde Pública. 2004; 38: $261-7$.

8. Motta GdCPd, Echer IC, LucenaAdF. Factors associated with smoking in pregnancy. Rev Latino-Am Enf. 2010; 18: 809-15.

9. Freire K, Padilha PdC, Saunders C. Fatores associados ao uso de álcool e cigarro na gestação. Rev Bras Ginecol probabilidade de fumar durante a gestação. A identificação das características locais associadas permitirá que as equipes de saúde planejem intervenções mais enfáticas às mulheres com fatores de vulnerabilidade, garantindo benefícios à saúde da mulher e reduzindo a morbimortalidade infantil relacionada ao uso do tabaco.

\section{Agradecimentos}

À equipe do grupo de pesquisa em Saúde Coletiva da Universidade Federal do Maranhão, que foram fundamentais para a coleta, análise de dados e confecção deste artigo. Agradecimentos ao Conselho Nacional de Desenvolvimento Científico e Tecnológico $(\mathrm{CNPq})$ pelo auxílio financeiro (bolsa de iniciação científica) ao longo do período de execução.
Obstetr. 2009; 31: 335-41

10. Reis LG, Silva CJd, Trindade A, Abrahão M, Silva VAd. Women who smoke and stop during pregnancy: who are they? Rev Bras Saúde Matern Infant. 2008; 8: 217-21.

11. Bezerra J, Barros MVG, Tenório MCM, Tassitano RM, Barros SSH, Hallal PC. Religiosidade, consumo de bebidas alcoólicas e tabagismo em adolescentes. Rev Panam Salud Publica. 2009; 26: 440-6.

12. Anthony D, Chowdary Q, Dyson P, Thankappan K. Does ethnicity or religion affect and/or explain the relationship between knowledge, attitudes and beliefs, and smoking behaviour? Divers Equality Health Care. 2013; 10: 31-40.

13. Takahasi EHM, Alves MTSSB, Alves GS, Silva AAM, Batista RFL, Simões VMF, Del-Ben CM, Barbieri MA. Mental health and physical inactivity during pregnancy: a cross-sectional study nested in the BRISA cohort study. Cad Saúde Pública. 2013; 29 (8): 1583-94.

14. Figuerêdo ED, Lamy FF, Lamy ZC, Silva AAM. Maternal age and adverse perinatal outcomes in a birth cohort (BRISA) from a Northeastern Brazilian city. Rev Bras Ginecol Obstet. 2014; 36 (12): 562-8.

15. ABEP (Associação Brasileira de Empresas de Pesquisa). Critério de Classificação Econômica Brasil: dados com base no levantamento sócio econômico 2005, IBOPE. 2008 [acesso em 25 out 2014]. Disponível em: http://www.abep. org.

16. Althabe F, Colomar M, Gibbons L, Belizan JM, Buekens P. Tabaquismo durante el embarazo en Argentina y Uruguay. Medicina (Buenos Aires). 2008; 68: 48-54.

17. Ekblad M, Gissler M, Korkeila J, Lehtonen L. Trends and risk groups for smoking during pregnancy in Finland and other Nordic countries. Eur J Public Health. 2014; 24 (4): 544-51. 
18. São Paulo (Estado). Secretaria da Saúde. Coordenadoria de Planejamento em Saúde. Assessoria Técnica em Saúde da Mulher. Atenção à gestante e à puérpera no SUS - SP: manual técnico do pré natal e puerpério. São Paulo, SP; 2010.

19. WHO (World Health Organization). MPOWER: a policy package to reverse the tobacco epidemic. Geneva; 2008.

20. Panjari M, Bell RJ, Astbury J, Bishop SM, Dalais F, Rice GE. Women who spontaneously quit smoking in early preg nancy. Aust N Z J Obstet Gynaecol. 1997; 37 (3): 271-8.

21. McLeod D, Pullon S, Cookson T. Factors that influence changes in smoking behaviour during pregnancy. $\mathrm{N} Z$ Med J. 2003; 116 (1173): U418

22. Pérez-Ríos M, Santiago-Pérez MI, Alonso B. Abandono del consumo de tabaco en las fumadoras gallegas durante el embarazo o la lactancia, 1954-2004. Gac Sanit. 2006; 20 (5): 392-5.

23. Dalgalarrondo P, Soldera MA, Corrêa Filho HR, Silva CAM. Religião e uso de drogas por adolescentes. Rev Bras Psiq. 2004; 26: 82-90.

24. Gomes FC, Andrade AG, Izbicki R, Moreira-Almeida A, Oliveira LG. Religion as a protective factor against drug use among brazilian university students: a national survey. Rev Bras Psiq. 2013; 35: 29-37.

Recebido em 16 de março de 2015

Versão final apresentada em 29 de junho de 2015

Aprovado em 01 de julho de 2015
25. Piccinini CA, Marin AH, Gomes AG, Lopes RCS. A constituição da maternidade em gestantes solteiras. Psico. 2011; 42: $246-54$

26. Torrent M, Sunyer J, Cullinan P, Basagana X, Harris J, Garcia O, Antó JM. Smoking cessation and associated factors during pregnancy. Gac Sanit. 2004; 18 (3): 184-9.

27. Jhun H-J, Seo H-G, Lee D-H, Sung M-W, Kang Y-D, Syn H C, Jun J K. Self-reported Smoking and Urinary Cotinine Levels among Pregnant Women in Korea and Factors Associated with Smoking during Pregnancy. J Korean Med Sci. 2010; 25 (5): 752-7.

28. Horta BL, Victora CG, Barros FC, Santos IS, Menezes AMB. Tobacco smoking among pregnant women in an urban area in southern Brazil, 1982 and 1993. Rev Public Health [online]. 1997; 31 (3): 247-53.

29. Menezes AMB, Minten GC, Hallal PC, Victora CG, Horta BL, Gigante DP, Barros FC. Tabagismo na coorte de nascimentos de 1982: da adolescência à vida adulta, Pelotas, RS Rev Saúde Pública. 2008; 42 (2): 78-85.

30. Rocha RS, Bezerra SC, Lima JWO, Costa FS. Consumo de medicamentos, álcool e fumo na gestação e avaliação dos riscos teratogênicos. Rev Gaúcha Enferm. 2013; 34 (2): 37 45 